\title{
Positive Solution for P-laplace Problems with Nonlinear Time-fractional Differential Equation
}

\author{
Shifeng Zhang* \\ Tourism and Culture College \\ Yunnan University \\ Lijiang, China \\ *Corresponding author \\ Zhiyang Jia \\ Tourism and Culture College \\ Yunnan University \\ Lijiang, China
}

\author{
Jihe Wang \\ Tourism and Culture College \\ Yunnan University \\ Lijiang, China \\ Qijiu Yang \\ Tourism and Culture College \\ Yunnan University \\ Lijiang, China
}

\begin{abstract}
In recent years, fractional differential equations are widely used in the many academic disciplines--viscoelastic mechanics, Fractal theory and so on. Furthermore, fractional differential equations can be used to describe some abnormal phenomenon. For instance, fractional convection-diffusion equation can be used to describe the fluid of abnormal infiltration phenomenon in the medium. In this paper, by means of the Arzela-Ascoli fixed point theorem, we can prove the existence of solution for the time-fractional differential equations. The conclusion is given out in detail.
\end{abstract}

Keywords-weighted sobolev space; time-fractional; caputo derivative; integral transform; fixed point theorem

\section{INTRODUCTION}

In the paper, we study the positive solution for following $\mathrm{p}$ Laplace problems with nonlinear time-fractional differential equation:

$$
\left\{\begin{array}{cc}
D^{\kappa} \mathrm{u}(\mathrm{x}, \mathrm{t})=\operatorname{div}\left(\mathrm{b}(\mathrm{x})|\mathrm{Du}(\mathrm{x}, \mathrm{t})|^{\mathrm{p}-2}|\mathrm{Du}(\mathrm{x}, \mathrm{t})|\right)+ \\
\mathrm{h}(\mathrm{x})|\mathrm{u}(\mathrm{x}, \mathrm{t})|^{\mathrm{r}-2} \mathrm{u}(\mathrm{x}, \mathrm{t})+\mathrm{f}(\mathrm{x}, \mathrm{u}) & \text { in } \Omega_{\mathrm{T},} \\
\mathrm{u}(\mathrm{x}, \mathrm{t})=0 & \text { on } \partial \Omega_{\mathrm{T}} \\
\mathrm{u}(\mathrm{x}, 0)=\varphi(\mathrm{x}) & \text { in } \Omega_{\mathrm{t}} \\
\mathrm{u}_{\mathrm{t}}(\mathrm{x}, \mathrm{t})=\psi(\mathrm{x}) & \text { in } \Omega .
\end{array}\right.
$$

Where $\Omega$ is a bounded domain with smooth boundary in $R^{n}, \Omega_{T}=\Omega \times[0, \mathrm{~T}], D^{\alpha}$ denotes the Caputo fractional derivatives [3], $1<\alpha<2$ is a parameter describing the order of the fractional time, and $\varphi(x), \psi(x) \in H_{0}^{1}(b(x), \Omega)$ are given real-valued functions. $b(x), h(x), f(x, u)$ are measurable functions and satisfy the following conditions

(H1) $0 \leq b(\mathrm{x}) \in \mathrm{L}^{\infty}(\Omega)$, and $\operatorname{satisfies}(\mathrm{x}) \rightarrow 1$ as $|x| \rightarrow \infty . b(s)$ is a positive weight function, Locally Hölder continous, and almost everywhere with positive measure in the Sobolev space $W_{0}^{1, p}(\mathrm{~b}(\mathrm{x}), \Omega)$ which with the standard norm

$$
\|\mathrm{u}\|=\left\{\int_{\Omega} \mathrm{b}(\mathrm{x})|\mathrm{Du}(\mathrm{x})|^{\mathrm{p}} \mathrm{dx}\right\}^{\frac{1}{\mathrm{p}}}
$$

(H2) $0 \leq \mathrm{h}(\mathrm{x}) \in \mathrm{l}^{2}(\Omega)$, and $h(x)$ has a compact support in $\Omega$,

(H3) $|f(x, u)| \leq C|u|^{(q-1)}, C>0$.

As we all know, the subject of fractional different equations has emerged as an important area of investigation. Indeed, we can find numbers applications in viscoelasticity, electrochemistry, control, electromagnetic, etc.

In consequence, the subject of fractional differential equations is gaining much importance and attention. However, there are few papers consider the p-Laplace problems with nonlinear partial differential equations of time-fractional order, as shown in $[3,9,10]$.

Consequently, the problem (1) is deduced to an equivalent integral equation under the fractional order integral operator $\mathrm{I}^{\wedge} \alpha$.At last, we proof the existence of solution for the timefractional differential equation by exploiting Arzela-Ascoli fixed point theorem.

\section{SOME IMPORTANT DEFINITIONS AND LEMMAS}

We can denote the Sobolev space $W_{0}^{1, p}(\Omega)$ by $X$. In addition, we study the fractional order equation (1) in the weighted Sobolev space $H_{0}^{1}(\mathrm{~b}(\mathrm{~s}), \Omega)$ which the standard norm

$$
\|u\|_{H_{0}^{1}(b(x), a)}=\left\{\int_{\Omega}(|b(x) D u|)^{2} d x\right\}^{\frac{1}{2}}
$$


To Discuss the existence of the positive solution for the equation(1), we present some basic notations, definitions , Lemmas and preliminary results which we will be used through the paper.

Definition 2.1 The Caputo fractional derivative of order $\alpha$ of a functionf $(\mathrm{t}), \mathrm{t}>0$, is defined as

$$
D^{\alpha} f(t)=\frac{1}{\Gamma(1-(\alpha))} \int_{0}^{t} \frac{1}{(t-s)^{(\alpha)}} f(t)^{([\alpha]+1)} d s
$$

Where in, $(\alpha),[\alpha]$ denotes the fractional and the integer part of $\alpha$ respectively, is the Gamma function.

Definition 2.2 The Riemann-Liouville fractional integral of order $\alpha$ of a function $f(t), t>0$, is defined as

$$
I_{0}^{\alpha}+f(t)=\frac{1}{\Gamma(\alpha)} \int_{0}^{t}(t-s)^{\alpha-1} f(s) d s
$$

The right side is point wise defined on .

Lemma 2.1 Assume that $y \in C[0, \mathrm{~T}], T>0,1<\alpha<2$, then the problem has the unique solution

$$
u(t)=u(0)+u(0) t+\frac{1}{\Gamma(\omega)} \int_{0}^{t}(t-s)^{\kappa-1} f(s) d s
$$

Now we establish some results of the existence of positive solution for the equation (1).

By Lemma 2.1, we may reduce the equation (1) to an equivalent integral equation as following:

$$
\begin{gathered}
\quad-\varphi(\mathrm{x})-\psi(\mathrm{x}) \mathrm{t}+\mathrm{u}(\mathrm{x}, \mathrm{t})= \\
\frac{1}{\Gamma(\alpha)} \int_{0}^{\mathrm{t}}(\mathrm{t}-\mathrm{s})^{\alpha-1}\left(\operatorname{div}\left(\mathrm{b}(\mathrm{x})|\mathrm{Du}(\mathrm{x}, \mathrm{s})|^{\mathrm{p}-2} \mathrm{Du}(\mathrm{x}, \mathrm{t})\right)\right. \\
+\mathrm{h}(\mathrm{x})|\mathrm{u}(\mathrm{x}, \mathrm{t})|^{\mathrm{r}-2} \mathrm{u}(\mathrm{x}, \mathrm{t})+\mathrm{f}(\mathrm{x}, \mathrm{u}(\mathrm{x}, \mathrm{s})) \mathrm{ds} \text { in } \Omega_{\mathrm{T}} \\
\mathrm{u}(\mathrm{x}, \mathrm{t})=0, \quad \text { on } \partial \Omega_{\mathrm{T}}
\end{gathered}
$$

$$
\text { (1, integral) }
$$

The theory of integral equations is developing rapidly with the help of the tools in functional analysis, topology and fixedpoint theory and it serves as a useful tool in turn for other branches of mathematics, for example for different equation. Now we define

$$
\begin{gathered}
\Phi(\mathrm{u})=\varphi(\mathrm{x})+\psi(\mathrm{x}) \mathrm{t}+ \\
\frac{1}{\Gamma(\alpha)} \int_{0}^{\mathrm{t}}(\mathrm{t}-\mathrm{s})^{\alpha-1}\left(\operatorname{div}\left(\mathrm{b}(\mathrm{x})|\mathrm{Du}(\mathrm{x}, \mathrm{s})|^{\mathrm{p}-2} \mathrm{Du}(\mathrm{x}, \mathrm{t})\right)\right. \\
+\mathrm{h}(\mathrm{x})|\mathrm{u}(\mathrm{x}, \mathrm{t})|^{\mathrm{r}-\mathrm{z}} \mathrm{u}(\mathrm{x}, \mathrm{t})+\mathrm{f}(\mathrm{x}, \mathrm{u}(\mathrm{x}, \mathrm{s})) \mathrm{d} \mathrm{s} \text { in } \Omega_{\mathrm{T}} \\
\mathrm{u}(\mathrm{x}, \mathrm{t})=0, \quad \text { on } \partial \Omega_{\mathrm{T}}
\end{gathered}
$$

Definition 2.3 We call $u \in C\left([0, \mathrm{~T}] ; \mathrm{H}_{0}^{1}(\mathrm{~b}(\mathrm{x}), \Omega)\right)$ to be a weak solution of the fractional order equation (1), if $\int_{\Omega}(\mathrm{u}-\Phi(\mathrm{u}) \mathrm{vdx}=0), \forall t \in[0, \mathrm{~T}]$ for evey $v \in H_{0}^{1}(\mathrm{~b}(\mathrm{x}), \Omega)$.

\section{A UNIQUE WEAK SOLUTION}

Now we proof the existence of a unique weak solution for problem.

Proposition

3.1 The operator $\Phi(\mathrm{u}): \mathrm{H}_{0}^{1}(\mathrm{~b}(\mathrm{x}), \Omega) \rightarrow \mathrm{H}_{0}^{1}(\mathrm{~b}(\mathrm{x}), \Omega)$ is completely continuous.

\section{Proof}

Putting

$$
\begin{aligned}
\mathrm{F}(\mathrm{u})= & \operatorname{div}\left(\mathrm{b}(\mathrm{x})|\mathrm{Du}(\mathrm{x}, \mathrm{s})|^{\mathrm{p}-2} \mathrm{Du}(\mathrm{x}, \mathrm{t})\right) \\
& +\mathrm{h}(\mathrm{x})|\mathrm{u}(\mathrm{x}, \mathrm{t})|^{\mathrm{r}-2} \mathrm{u}(\mathrm{x}, \mathrm{t})+\mathrm{f}(\mathrm{x}, \mathrm{u}(\mathrm{x}, \mathrm{s}))
\end{aligned}
$$

We can rewrite:

$$
\Phi(\mathrm{u})=\varphi(\mathrm{x})+\psi(\mathrm{x}) \mathrm{t}+\frac{1}{\Gamma(\omega)} \int_{0}^{t}(\mathrm{t}-\mathrm{s})^{\kappa-1} \mathrm{~F}(\mathrm{u}) \mathrm{ds}
$$

For each $v \in H_{0}^{1}(\mathrm{~b}(\mathrm{x}), \Omega)$, and $\|v\|_{H_{0}^{1}(\mathrm{~b}(\mathrm{x}), \Omega)}=1$, integration by parts, we can get:

$$
|<F(\mathrm{u}), v>|=\left.\left|\int \mathrm{b}(\mathrm{x})\right| \mathrm{Du}\right|^{\mathrm{p}-1} \mathrm{Dv}+\mathrm{h}(\mathrm{x})|\mathrm{u}|^{\mathrm{r}-\mathrm{z}} \mathrm{uv}+\mathrm{f}(\mathrm{x}, \mathrm{u}) \mathrm{vdx} \mid
$$

Since $0 \leq h(\mathrm{x}) \in \mathrm{L}^{2}(\Omega)$, and $h(x)$ has a compact support in $\Omega, b(\mathrm{x}) \in \mathrm{L}_{+}^{\infty}(\Omega)$, so

$$
F(u) \in C\left([0, \mathrm{~T}] ; \mathrm{H}_{0}^{1}(\mathrm{~b}(\mathrm{x}), \Omega)\right)
$$

And since $p>2$, by Sobolev imbedding theorem, we have

$$
\mathrm{W}_{\mathrm{Q}}^{1, \mathrm{p}}(\mathrm{b}(\mathrm{x}), \Omega) \rightarrow \mathrm{H}_{0}^{1}(\mathrm{~b}(\mathrm{x}), \Omega)
$$

Then, we can get

$$
\|\mathrm{u}\|_{\mathrm{H}_{0}^{1}(\mathrm{~b}(\mathrm{x}), \Omega)} \leq\|\mathrm{u}\|_{\mathbb{W}_{0}^{1}{ }^{1 \mathrm{p}}(\mathrm{b}(\mathrm{x}), \Omega)}
$$

And, we can denote $\|u\|_{H_{0}^{1}(\mathrm{~b}(\mathrm{x}), \Omega)}$ and $\|u\|_{H_{0}^{-1}(\mathrm{~b}(\mathrm{x}), \Omega)}$ by $\|u\|_{H_{0}^{1}}$, $\|u\|_{H_{0}^{-1}}$ respectively. Hence, by Cauchy-Schwarz inequalities, Poincare inequalities, Hölder inequalities, Sobolev imbedding theorm and $1<p<r$, we have 
$\leq\|\mathrm{Du}\|_{\mathrm{L}^{2(\mathrm{P}-1)}(\mathrm{b}(\mathrm{x}), \Omega)}^{\mathrm{p}-1}+\|\mathrm{h}(\mathrm{x})\|_{\mathrm{L}^{2}}\|\mathrm{u}\|_{\mathrm{L}^{(2 \mathrm{r}-1)}(\Omega)}^{\mathrm{r}-1}+\mathrm{C}\|\mathrm{u}\|_{\mathrm{L}^{2(q-1)}(\Omega)}^{\mathrm{q}-1}$

$\leq\|\mathrm{u}\|_{\mathrm{H}_{0}^{1}}^{\mathrm{p}-1}+\|\mathrm{h}(\mathrm{x})\|_{\mathrm{L}^{2}}\|\mathrm{u}\|_{\mathrm{L}^{2(r-1)}(\mathrm{b}(\mathrm{x}), \Omega)}^{\mathrm{r}-1}+\mathrm{C}\|\mathrm{u}\|_{\mathrm{L}^{2(q-1)}(\mathrm{g}(\mathrm{x}), \Omega)^{+}}^{\mathrm{q}-1}$

$\leq\|\mathrm{u}\|_{\mathrm{H}_{0}^{1}}^{\mathrm{p}-1}+\|\mathrm{h}(\mathrm{x})\|_{\mathrm{L}^{2}}\|\mathrm{Du}\|_{\mathrm{L}^{2(\mathrm{r}-1)}(\mathrm{b}(\mathrm{x}), \Omega)}^{\mathrm{r}-1}+\mathrm{C}\|\mathrm{Du}\|_{\mathrm{L}^{2(q-1)}(\mathrm{b}(\mathrm{x}), \Omega)^{4}}^{\mathrm{q}-1}$

$\leq\|\mathrm{u}\|_{\mathrm{H}_{0}^{1}}^{\mathrm{p}-1}+\|\mathrm{h}(\mathrm{x})\|_{\mathrm{L}^{2}}\|\mathrm{u}\|_{\mathrm{H}_{0}^{1}}^{\mathrm{r}-\mathrm{C}} \mathrm{C}\|\mathrm{u}\|_{\mathrm{H}_{0}^{1}}^{\mathrm{q}-1}$

$\leq\|\mathrm{u}\|_{\mathrm{X}}^{\mathrm{p}-1}+\|\mathrm{h}(\mathrm{x})\|_{\mathrm{L}^{2}}\|\mathrm{u}\|_{\mathrm{X}}^{\mathrm{r}-1}+\mathrm{C}\|\mathrm{u}\|_{\mathrm{X}}^{\mathrm{q}-1}$

$\leq\|\mathrm{Du}\|_{\mathrm{L}^{2}(\mathrm{P}-1)(\mathrm{b}(\mathrm{x}), \Omega)}^{\mathrm{p}-1}+\|\mathrm{h}(\mathrm{x})\|_{\mathrm{L}^{2}}\|\mathrm{u}\|_{\mathrm{L}^{2(\mathrm{r}-1)}(\Omega)}^{\mathrm{r}-1}+\mathrm{C}\|\mathrm{u}\|_{\mathrm{L}^{2(\mathrm{q}-1)}(\Omega)}^{\mathrm{q}-1}$

$\leq\|\mathrm{u}\|_{\mathrm{H}_{0}^{1}}^{\mathrm{p}-1}+\|\mathrm{h}(\mathrm{x})\|_{\mathrm{L}^{2}}\|\mathrm{u}\|_{\mathrm{L}^{2(\mathrm{r}-1)}(\mathrm{b}(\mathrm{x}), \Omega)}^{\mathrm{r} r-1}+\mathrm{C}\|\mathrm{u}\|_{\mathrm{L}^{2(\mathrm{q}-1)}(\mathrm{b}(\mathrm{x}), \Omega)^{4}}^{\mathrm{q}-1}$

$\leq\|\mathrm{u}\|_{\mathrm{H}_{0}^{1}}^{\mathrm{p}-1}+\|\mathrm{h}(\mathrm{x})\|_{\mathrm{L}^{2}}\|\mathrm{Du}\|_{\mathrm{L}^{2(\mathrm{r}-1)}(\mathrm{b}(\mathrm{x}), \Omega)}^{\mathrm{r}-1}+\mathrm{C}\|\mathrm{Du}\|_{\mathrm{L}^{2}(\mathrm{q}-1)}^{\mathrm{q}-1}(\mathrm{~b}(\mathrm{x}), \Omega)^{4}$

$\leq\|\mathrm{u}\|_{\mathrm{H}_{0}^{1}}^{\mathrm{p}-1}+\|\mathrm{h}(\mathrm{x})\|_{\mathrm{L}^{2}}\|\mathrm{u}\|_{\mathrm{H}_{0}^{1}}^{\mathrm{r}-1} \mathrm{C}\|\mathrm{u}\|_{\mathrm{H}_{0}^{1}}^{\mathrm{q}-1}$

$\leq\|\mathrm{u}\|_{\mathrm{X}}^{\mathrm{p}-1}+\|\mathrm{h}(\mathrm{x})\|_{\mathrm{L}^{2}}\|\mathrm{u}\|_{\mathrm{X}}^{\mathrm{r}-1}+\mathrm{C}\|\mathrm{u}\|_{\mathrm{X}}^{\mathrm{q}-1}$

From [4], we know that $u \in X$ is bounded, that is to say, $\|u\|_{x} \leq M(M$ is a constant $)$.Then,

$$
\|\mathrm{u}\|_{\mathrm{X}}^{\mathrm{p}-1}+\|\mathrm{h}(\mathrm{x})\|_{\mathrm{L}^{2}}\|\mathrm{u}\|_{\mathrm{X}}^{\mathrm{r}-1}+\mathrm{C}\|\mathrm{u}\|_{\mathrm{X}}^{\mathrm{q}-1} \leq \mathrm{M}_{1}
$$

Thus, by Cauchy-Schwarz inequalities, we obtain

$$
\begin{aligned}
& \|\Phi(\mathrm{u})\|_{\mathrm{H}_{0}^{-1}={ }_{\|u\|_{\mathbf{H}_{0}} \leq 1} \sup }|\langle\phi(\mathrm{u}), \mathrm{U}\rangle|
\end{aligned}
$$

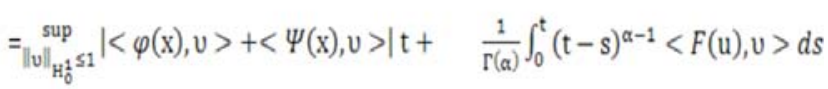

$$
\begin{aligned}
& \leq|\langle\varphi(x), v\rangle| \text {. } \\
& +|\langle\Psi(\mathrm{x}), \mathrm{v}\rangle \mathrm{t}|+\left|\frac{1}{\Gamma(\alpha)} \int_{0}^{\mathrm{t}}(\mathrm{t}-\mathrm{s})^{\alpha-1}\langle F(\mathrm{u}), \mathrm{u}\rangle d s\right| . \\
& \left.\leq\|\varphi(x)\|_{L^{\infty}(b(x), \Omega)}\|v\|_{H_{0}^{1}}+\| \Psi(x)\right)\left\|_{L^{\infty}(b(x), \Omega)}\right\| v \|_{H_{0}^{1}} \\
& +|<F(\mathrm{u}), \mathrm{v}\rangle|| \frac{1}{\Gamma(\alpha)} \int_{0}^{\mathrm{t}}(\mathrm{t}-\mathrm{s})^{\alpha-1} \mathrm{ds} \mid
\end{aligned}
$$$$
\left.\leq\|\varphi(x)\|_{L^{\infty}(b(x), \Omega)}+\| \Psi(x)\right) \|_{L^{\infty}(b(x), \Omega)}+\frac{M_{1}}{\Gamma(\alpha)}\left|\int_{0}^{t}(t-s)^{\alpha-1} d s\right|
$$$$
\left.\leq\|\varphi(x)\|_{L^{\infty}(b(x), \Omega)}+\| \Psi(x)\right) \|_{L^{\infty}(b(x), \Omega)}+\frac{M_{1} t^{\alpha}}{\alpha \Gamma(\alpha)}
$$$$
\left.\leq\|\varphi(x)\|_{L^{\infty}(b(x), \Omega)}+\| \Psi(x)\right) \|_{L^{\infty}(b(x), \Omega)}+\frac{M_{1} T^{\alpha}}{\alpha \Gamma(\alpha)}
$$

Hence, $\Phi(\mathrm{u})$ is bounded.

On the other hand, given $\varepsilon>0$, setting

$$
\delta=\left\{\left(\|\Psi(\mathrm{x})\|_{L^{\infty}(\mathrm{b}(\mathrm{x}), \Omega)}+\frac{\mathrm{M}_{1}}{\Gamma(\mathrm{w})}\right)^{-1} \varepsilon\right\}^{\frac{1}{\alpha}}
$$

That is to say, $\Phi(\mathrm{u})$ is of equicontinuity; in fact

$$
\begin{aligned}
& \left\|\Phi \mathrm{u}\left(\mathrm{t}_{2}\right)-\Phi \mathrm{u}\left(\mathrm{t}_{1}\right)\right\|_{\mathrm{H}_{0}^{1}} \\
& =\sup _{\|u\|_{\mathrm{H}_{0} \leq 1} \leq 1}\left|\left\langle\varphi\left(\mathrm{t}_{2}\right)-\Phi u\left(\mathrm{t}_{1}\right), v\right\rangle\right| \\
& = \\
& \sup _{\|v\|_{H_{0}^{1} \leq 1} \mid} \mid\langle\Psi(\mathrm{x}), \mathrm{v}\rangle\left(\mathrm{t}_{2}-\mathrm{t}_{1}\right)+ \\
& \frac{1}{\Gamma(\alpha)} \int_{0}^{t_{2}}\left(t_{2}-s\right)^{\alpha-1}\left\langle F(u), u>d s-\frac{1}{\Gamma(\alpha)} \int_{0}^{t_{1}}\left(t_{1}-s\right)^{\alpha-1}\langle F(u), v>d s|\right. \\
& \leq\|\Psi(\mathrm{x})\|_{L^{\infty}(\mathrm{b}(\mathrm{x}), \Omega)}\|v\|_{\mathrm{H}_{0}^{1}}\left|\mathrm{t}_{2}-\mathrm{t}_{1}\right|+|<F(\mathrm{u}), \mathrm{v}>|\left|\frac{1}{\Gamma(\alpha)} \int_{0}^{\mathrm{t}_{2}}\left(\mathrm{t}_{2}-\mathrm{s}\right)^{\alpha-1} d s\right| \\
& -|\langle F(\mathrm{u}), \mathrm{v}\rangle|\left|\frac{1}{\Gamma(\alpha)} \int_{0}^{\mathrm{t}_{1}}\left(\mathrm{t}_{1}-s\right)^{\alpha-1} d s\right| .
\end{aligned}
$$

$\leq\|\Psi(x)\|_{L^{\infty}(b(x), \Omega)}\left|t_{2}-t_{1}\right|+\frac{M_{1}}{\Gamma(\alpha)} \frac{t_{2}^{\alpha}}{\alpha}-\frac{M_{1}}{\Gamma(\alpha)} \frac{t_{1}^{\alpha}}{\alpha}$

$=\|\Psi(\mathrm{x})\|_{L^{\infty}(b(x), \Omega)}\left|t_{2}-t_{1}\right|+\frac{M_{1}}{\alpha \Gamma(\alpha)}\left(t_{2}^{\alpha}-t_{1}^{\alpha}\right)$.

Then we divide the proof into two cases

Case 1: $\delta \leq t_{1} \leq t_{2}$. Since $1<\alpha<2$, we can get

$$
\begin{aligned}
& \left\|\Phi u\left(t_{2}\right)-\Phi u\left(t_{1}\right)\right\|_{H_{0}^{1}}=\sup _{\|v\|_{H_{0}^{1}} \leq 1} \mid\left\langle\Phi u\left(t_{2}\right)-\Phi u\left(t_{1}\right), v\right\rangle \| \\
& \leq\|\Psi(x)\|_{L^{\infty}(b(x), \Omega)}\left|t_{2}-t_{1}\right|+\frac{M_{1}}{\alpha \Gamma(\alpha)}\left(t_{2}^{\alpha}-t_{1}^{\alpha}\right) \\
& =\|\Psi(x)\|_{L^{\infty}(b(x), \Omega)}\left|t_{2}-t_{1}\right|+\frac{M_{1}}{\alpha \Gamma(\alpha)} t^{\alpha}\left(t_{2}-t_{1}\right) . \\
& \leq\|\Psi(x)\|_{L^{\infty}(b(x), \Omega)}\left|t_{2}-t_{1}\right|+\frac{M_{1}}{\Gamma(\alpha)} \frac{1}{\delta^{(1-\alpha)}}\left(t_{2}-t_{1}\right) . \\
& \leq\|\Psi(x)\|_{L^{\infty}(b(x), \Omega)} \delta+\frac{M_{1}}{\Gamma(\alpha)} \delta^{\alpha} . \\
& \leq\|\Psi(x)\|_{L^{\infty}(b(x), \Omega)} \delta^{\alpha}+\frac{M_{1}}{\Gamma(\alpha)} \delta^{\alpha} . \\
& =\left(\|\Psi(x)\|_{L^{\infty}(b(x), \Omega)}+\frac{M_{1}}{\Gamma(\alpha)}\right) \delta^{\alpha} \leq \varepsilon_{.}
\end{aligned}
$$

Case 2: When $0<t_{1}, t_{2}<\alpha^{\frac{1}{\alpha}} \delta$, we can get 


$$
\begin{aligned}
& \left\|\Phi u\left(t_{2}\right)-\Phi u\left(t_{1}\right)\right\|_{H_{0}^{1}} . \\
& =\sup _{\|v\|_{H_{1}^{1} \leq 1}}\left|\left\langle\Phi u\left(t_{2}\right)-\Phi u\left(t_{1}\right), v\right\rangle\right| \\
& \leq\|\Psi(x)\|_{L^{\infty}(b(x), \Omega)}\left|t_{2}-t_{1}\right|+\frac{M_{1}}{\alpha \Gamma(\alpha)}\left(t_{2}^{\alpha}-t_{1}^{\alpha}\right) . \\
& \leq\|\Psi(x)\|_{L^{\infty}(b(x), \Omega)} \delta+\frac{M_{1}}{\alpha \Gamma(\alpha)} \alpha \delta^{\alpha} . \\
& \leq\|\Psi(x)\|_{L^{\infty}(b(x), \Omega)} \delta^{\alpha}+\frac{M_{1}}{\Gamma(\alpha)} \delta^{\alpha} . \\
& =\left(\|\Psi(x)\|_{L^{\infty}(b(x), \Omega)}+\frac{M_{1}}{\Gamma(\alpha)}\right) \delta^{\alpha} \leq \varepsilon .
\end{aligned}
$$

By the means of the Arzela-Ascoli theorem, we know that: $\Phi(\mathrm{u}): \mathrm{H}_{0}^{1}(\mathrm{~b}(\mathrm{~s}), \Omega) \rightarrow \mathrm{H}_{0}^{1}(\mathrm{~b}(\mathrm{x}), \Omega)$ is completely continuous. This completes the proof.

By proposition 3.1, we know that $\int_{\Omega}(\mathrm{u}-\Phi(\mathrm{u})) \mathrm{vdx}=0$ and $\forall t \in[0, \mathrm{~T}]$ for every $v \in H_{0}^{1}(\mathrm{~b}(\mathrm{x}), \Omega)$, that is to say , the fractional order equation(1) has a unique weak solution $u \in C\left([0, \mathrm{~T}] ; \mathrm{H}_{0}^{1}(\mathrm{~b}(\mathrm{x}) \Omega)\right)$

\section{ACKNOWLEDGEMENT}

The research work was supported by Foundation of Tourism and Culture College of Yunnan University under Grant No. 2013XY15.

\section{REFERENCES}

[1] P. Drabek, A. kufner and F. Nicolosi, Quasilinear elliptic with degenerations and singularities [M], Walter de Gruyter. Berlin, New York, USA, 1997.

[2] R. A. A dams and J. F. F. John, Sobolev space [M], Acad. Press, New York, USA, 2009.

[3] M. L. Qiu, M. L. Quan, G. S. Yang, Positive solutions for p-Laplace problems with nonlinear time-fractional differential equation.

[4] S. F. Zhang, G. S. Yang, pairs of positive solutions of P-Laplace equations. Math. Sci. Res. J. 15(2011), no. 1, 12-19.

[5] L. Damascelli, M. Grossi, F. Pacella, Qualitative ptoperties of positive solutions of semilinear elliptic equations in symmetric domains via the maximum principle, J. Ann. Inst . H. Poincare Anal. Non., 16(1999)631652.

[6] K. S. Miller, B. Ross, An introduction to the fractional calculus and fractional differential equations [M], Wiley, New York, 1993.

[7] I. Podlubny, Fractional differential equations [M], Math. Sci. and Engineering, Acad. Press, New York, 1999.

[8] S. G. Samko, A. A. Kilbas, O. I. Marichev, Fractional integrals and derivatives, theory and applications [M], Gordon and Breach, Yverdon, 1993.

[9] A. A. Kilbas, H. M. Srivastava, J. J. Trujillo, Theory and applications of fractional differential equations [M], Elsevier, Amsterdam, 2006.

[10] A. A. Kilbas, J. J. Trujillo, Differential equations of fractional order: methods, results and problems, II, Appl. Anal., 81(2002) 435 C493. 